\title{
Decentralized Voltage Control for Efficient Power Exchange in Interconnected DC Clusters
}

\author{
Cephas Samende, Student Member, IEEE, Fei Gao Member, IEEE, Sivapriya M. Bhagavathy, \\ Member, IEEE, Malcolm McCulloch, Senior Member, IEEE
}

\begin{abstract}
Interconnection of two autonomous swarm grids (DC clusters) which are designed for energy access can increase power supply availability for power hungry appliances such as water pumps. Here, a surplus or a deficit power in one cluster is balanced by a second cluster through power exchange. Usually, the clusters are connected by a tie line if bus voltages in both clusters are equal and constant. If the bus voltages are different and constant, a single converter is used to interconnect the clusters and to control the power flow between them. In both interconnections, power flow is triggered by bus voltage variations from their constant values. However, if the bus voltages are different and are not constant, the coordination of the power exchange between the clusters is a challenge. In this paper, two buck-boost converters are proposed to interconnect the clusters that have different and unregulated bus voltages. To control the power exchange while reducing transmission line loss, a decentralized control approach which is composed of a voltage droop control method and a mid-point voltage control method is proposed. Simulations of two interconnected clusters carried out in MATLAB/Simulink software show how the proposed control method can efficiently coordinate the power exchange.
\end{abstract}

Index Terms-Interconnected swarm grids, DC clusters, voltage droop, mid-point voltage control, transmission line loss, energy access.

\section{INTRODUCTION}

$\mathrm{I}^{\mathrm{N}}$ $\mathrm{N}$ this paper, the challenge of efficiently coordinating power exchange between two autonomous DC clusters which are designed for energy access in off-grid communities of SubSaharan Africa (SSA) is addressed.

Currently, one out of seven people in the world still live without access to energy [1]. About two-thirds of them live in SSA, mainly in the rural areas [2]. As a result, they lack access to energy-enabled productive activities such as egg incubation, and water pumping system for irrigation which can generate income and alleviate poverty. The SDG 7 tracking report 2019 [2] reports that with the current political will, population growth and financial commitments, other regions of the world except SSA are likely to achieve the energy access target by 2030; it will take SSA up to 2080 to achieve the same energy access target, mainly due to the population growth which is projected to grow by half a billion in 2040 [3], [4]. Therefore,

\footnotetext{
${ }^{\mathrm{T}}$ his work was supported by the Rhodes Trust under Rhodes Scholarship.

C. Samende, S. M. Bhagavathy and M. McCulloch are with the Department of Engineering Science, Energy and Power Group, University of Oxford, Oxford, UK (e-mail: cephas.samende, sivapriya.mothilalbhagavathy, malcolm.mcculloch@eng.ox.ac.uk).Fei Gao is with Shanghai Jiao Tong University, Shanghai, China (e-mail: fei.gao@sjtu.edu.cn).
}

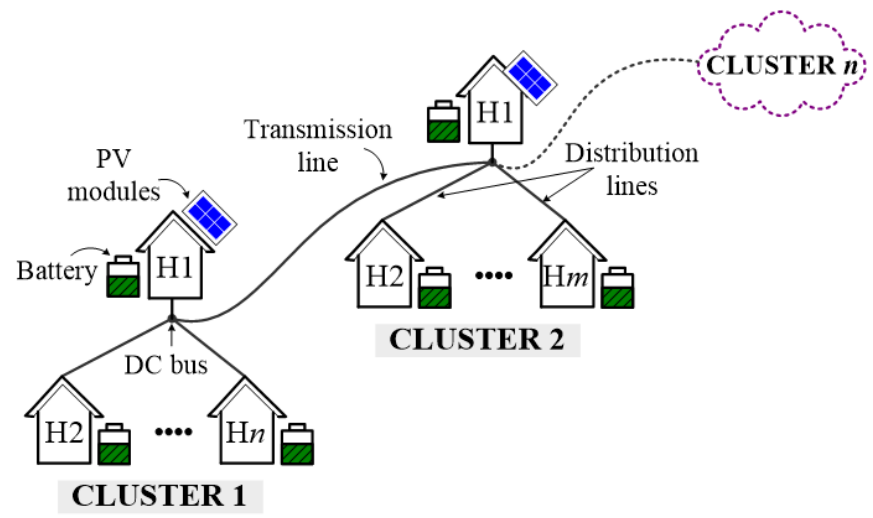

Fig. 1. Interconnection of clusters in off-grid communities for energy access.

robust electrification efforts especially those targeting populations in rural areas of SSA are required [5].

Given that the average population density of SSA is generally low, about 51 people $/ \mathrm{km}^{2}$ (equivalent to $10-20$ homes $/ \mathrm{km}^{2}$ ) [6], one of the most cost-effective and innovative approaches for providing energy access for productive use to the rural areas is the swarm electrification approach [7]. Here, homes which have a power electronics converter and a battery energy storage system (BESS), a solar photovoltaic (PV) module(s) or a combination of PV and BESS forming what is widely known as a solar home system (SHS) [8] are gradually interconnected to form a diverse peer-to-peer (P2P) grid (herein referred to as a DC cluster) as shown in Fig. 1. The DC cluster can operate autonomously and can after some time be expanded towards a national grid by interconnecting with other DC clusters and eventually interconnect with the national grid [9].

This kind of bottom-up approach supports the exchange of energy between homes and between clusters, thereby leveraging the inherent energy surplus which is not utilized in individual SHSs [8]. The sharing of the energy generation and storage resources through power exchange can enable homes to: (1) run power hungry appliances such as welding machines for productive uses without having to increase the power capacity of the existing SHS [8], and (2) gain access to energy without having to acquire a SHS [10], thereby accelerating the energy access progress at affordable costs.

In this paper, the operation of two interconnected clusters; cluster 1 and cluster 2 which are shown in Fig. 1 and which are not connected to a national grid is investigated. The transmission line is considered to be long, (e.g. in the range of 
$300 \mathrm{~m}$ to $2 \mathrm{~km}$ ), which is typical of the distance between villages (where a cluster is viewed to service a single village) in many rural areas of SSA due to hills, streams and farm blocks. For safety reasons, a nominal DC voltage of $120 \mathrm{~V}$ and $150 \mathrm{~V}$ is considered for the distribution lines and the transmission line (TL) respectively. Due to the low DC voltages and the long TL, this means that the TL loss is significant during power exchange.

Furthermore, to reduce cabling costs and for galvanic isolation, each home in the clusters shown in Fig. 1 uses a four port DC-DC converter (FPC) [11] to manage the power flow on the distribution line, the BESS, the appliances, and/or the PV modules. To control the power flow on the distribution line, the FPC adjusts the distribution voltage local to a home based on the power requirements of that home [12]. Power exchange between the homes in each cluster is therefore intuitively through voltage difference. Due to the variable distribution voltages, the voltage at the DC bus which is referred to as the DC bus voltage is also variable and can be different in both clusters at each time interval. This makes the interconnection of the clusters and the coordination of power exchange between them a challenge.

There are two ways reported in literature that can be used to interconnect the clusters and to coordinate the power exchange between them. The first and straightforward way is to directly connect the clusters using a tie line as proposed in [13], [14]. This approach does not require a dedicated converter to control the power exchange. Thus, cost and conversion losses are reduced. However, the approach requires both clusters to have the same operating DC bus voltage so that any mismatch in the voltages can trigger the power exchange. Decentralized and hierarchical control approaches are proposed in [13] and in [14], [15] respectively to achieve the constant bus voltage levels, thereby controlling the power exchange between the clusters. However, for clusters shown in Fig. 1 where the DC bus voltages are different and variable in both clusters, the tie line approach and the associated control methods may not be suitable due to unnecessary power flow on the tie line which can cause unnecessary TL loss.

The second approach involves interconnecting the clusters through a dedicated single interlinking converter (IC) unit [16]-[26]. The interlink converter can be of either a bidirectional buck-boost converter type [16]-[22] or in certain applications which require galvanic isolation, a dual active bridge converter type [23]-[26]. Due to the IC on the TL, there is an added advantage of power flow control between the clusters and clusters with different operating DC bus voltage levels can be interconnected. Various control strategies have been proposed in [16]-[26] to control the power exchange via the IC. However, they all have the following features in common:

- Conventional DC microgrids with regulated DC bus voltages are considered. Thus, a single IC is appropriate in such applications to mitigate the bus voltage variations, thereby controlling the power exchange between the clusters.

- In applications where the TL is long, the single IC is either placed near one DC microgrid [22], [26], or in the middle of the TL [25], [18]. Thus, only the TL voltage at the terminal(s) of the IC can be adjusted while the TL voltage at the other end of the TL cannot be adjusted. However, to minimize the TL loss, both $\mathrm{TL}$ voltages at the ends of the TL are required to be adjustable. Thus, a single IC cannot be used to minimize the TL loss where the TL is long.

In this paper, two bidirectional buck-boost ICs are proposed to interconnect two nearby clusters shown in Fig. 1. Due to the TL which is long and the DC bus voltage which is variable and different in both clusters, the two clusters cannot be connected by either a tie line or a single IC. To avoid communication links for reliability and cost reasons due to the long TL, a decentralize control approach which is composed of a voltage droop control method and a mid-point voltage control method is proposed to control the power exchange between the clusters. The proposed approach takes advantage of the variable DC bus voltage in each cluster to schedule the power exchange on the TL. Therefore, explicit determination of the power surplus/deficit in a cluster which may be required to schedule a power exchange, and which would rather require a centralized controller to be determined is avoided. The main contributions of this paper are summarized as follows:

1) A voltage-voltage droop characteristic is proposed to automatically detect an overload/underload operating mode of a cluster and schedule an appropriate power exchange with a neighboring cluster. The method is decentralized and suitable for rural areas where centralized methods which relies on communication links are difficult to maintain and are often unreliable.

2) A mid-point voltage control method is proposed to reduce the TL loss where the TL is long. The method is only activated when one cluster is neither overloaded nor underloaded (i.e. the cluster can either import or export power). When a cluster is either overloaded or underloaded (i.e. the cluster has a specific amount of power deficit/surplus), minimizing the TL loss is not necessary and the power exchange between the clusters is managed by the voltage-voltage droop characteristic.

The two proposed approaches mentioned above can effectively coordinate the power exchange between the clusters while minimizing the TL loss.

The rest of the paper is organized as follows. Section II describes the operation and control of a single cluster. Section III describes the proposed control strategy for coordinating the power exchange between the clusters. Section IV describes the implementation of the proposed control strategy. Simulation results that verify the performance of the proposed strategy are given in Section V. Section VI concludes the paper.

\section{OPERATION AND CONTROL OF A CLUSTER}

This section describes the operation and control of a single cluster. Firstly, the power management in a home is described. Then, the power management in a cluster is presented.

\section{A. Power Management in a Home}

Fig. 2 shows a schematic of a single cluster (e.g. cluster $j$ ) 


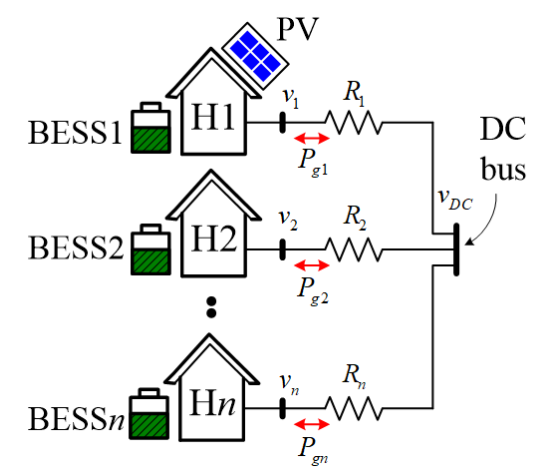

Fig. 2. Schematic of a single cluster with unregulated DC bus voltage.

considered in this paper. The cluster consists of multiple homes, $\mathrm{Hi}, i=1, \cdots, n$ as described in Section I. Each home uses a FPC to integrate the PV (which is optional in some homes), BESS, DC loads and to connect to other homes in the cluster via a distribution line as shown in Fig. 3. The principle of operation of the FPC is given in our previous work in [11]. In the homes without PV, the PV port on the FPC can be left either open or connected to other nearby homes. Advantages of using the FPC include reduced installation space requirements, and reduced cabling costs. The high frequency transformer (HFT) in the FPC also helps to provide different voltage levels and galvanic isolation between the homes [11]. The components of the FPC: (1) full bridge converters (FBC) denoted by $\mathrm{FBC} 1, \mathrm{FBC} 2$ and $\mathrm{FBC} 3$ and (2) half bridge converters (HBC) denoted by $\mathrm{HBC} 1, \mathrm{HBC} 2, \mathrm{HBC} 3$ and HBC4 in Fig. 3 are independently controlled. Thus, cheap control strategies and controllers such as the proportionalintegral (PI) controllers are designed to manage the power flow in the home(s).

The FBCs are phase shift, $\phi$ controlled while the HBCs are duty cycle, $d$ controlled [11]. The PV is operated in MPPT mode by controlling the HBC3 as shown in Fig. 4(a). $i_{p v}$ and $v_{p v}$ are measured terminal PV current and voltage respectively. The reference voltage, $v_{p v}{ }^{r e f}$ is generated by a classical MPPT algorithm [27]. The PI controllers provide the right $d$ to a pulse width modulator (PWM).

The FBC1 is taken as a reference $\mathrm{FBC}$ and is not controlled. The FBC2 and $\mathrm{FBC} 3$ are phase shift controlled and maintains a constant $38 \mathrm{~V}$ across $C_{2}$ and $152 \mathrm{~V}$ across $C_{3}$ respectively [11]. A generic control law of $\mathrm{FBC} 2$ and $\mathrm{FBC} 3$ is shown in Fig. 4(b). Since most DC loads in rural areas such as LED lights operate at a constant voltage, $\mathrm{HBC} 2$ is controlled to maintain a constant voltage of $12 \mathrm{~V}$. During local overloads, the power deficit is supplied to the loads by the BESS. Similarly, during local underloads, the excess PV generation is absorbed by the BESS. Thus, each home can manage its own generation and load demand autonomously provided the BESS are not over charged/discharged. The BESS is managed by controlling the $\mathrm{HBC} 1$ to maintain a constant $19 \mathrm{~V}$ across $C_{1}$. Fig. 5(a) shows the generic control law of both the DC loads and BESS.

\section{B. Power Management in a Cluster}

However, if the BESS in a home is either overcharged or

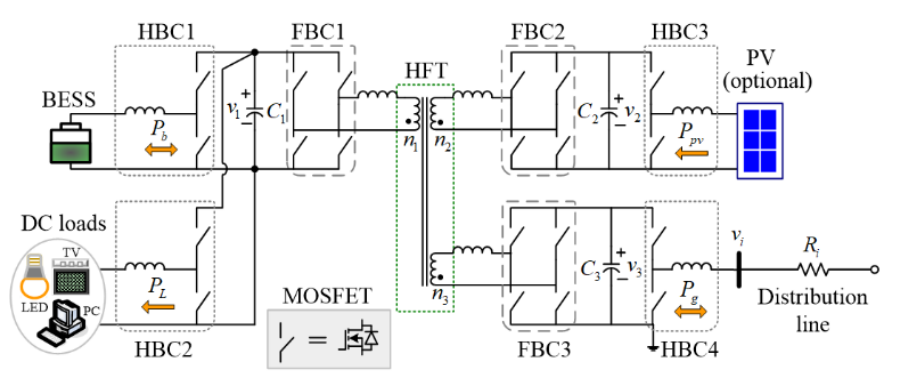

Fig. 3. Four port DC-DC converter (FPC) structure: Integration of PV (optional), BESS, DC loads and distribution line using a FPC in a home.

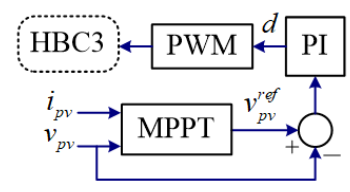

(a)

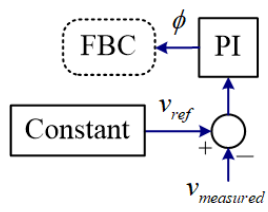

(b)
Fig. 4. Control loops of (a) the PV panel and (b) FBC2 and $\mathrm{FBC} 3$ in a home.

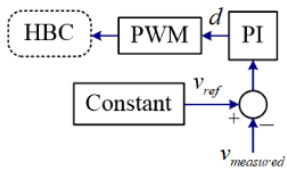

(a)

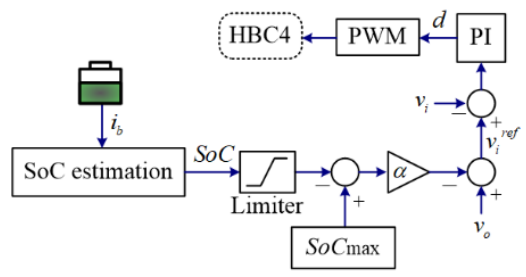

(b)
Fig. 5. Control loops of (a) BESS and DC loads and (b) the distribution line.

over discharged, the power surplus or power deficit can be managed by other homes in the cluster. Thus, the benefit of interconnecting homes in a cluster is to increase power supply reliability and to avoid unnecessary load shedding and PV curtailment. However, load shedding and PV curtailment may still be required when there is a power surplus or a power deficit in a home that other neighboring homes may fail to manage. Load shedding and PV curtailment techniques are however beyond the scope of this paper.

To control the power flow between the homes, each home sets its own distribution voltage $v_{i}$ based on its power requirements by controlling $\mathrm{HBC} 4$. In our previous work in [12], the reference distribution voltage, $v_{\text {ref }, i}$ required by HBC4 to set $v_{i}$ was determined based on the state of charge (SoC), $S o C_{i}$ of the local BESS as follows

$$
v_{\text {ref }, i}=v_{o}-\alpha\left(S o C_{\max }-S o C_{i}\right)
$$

where $v_{o}$ is nominal distribution voltage, $\alpha$ is droop gain, $S o C_{\max }$ is maximum SoC, $S o C_{i}$ is the $\mathrm{SoC}$ at time, $t$ which is estimated as $S_{o} C_{i}=\operatorname{SoC}_{o, i^{-}} \eta_{b, i} i_{b, i} \Delta t / C_{b, i}$, where $C_{b, i}$ is the BESS capacity, $\eta_{b, i}$ is the BESS charge/discharge efficiency, $i_{b, i}$ is the BESS output current and $\mathrm{SoC}_{o, i}$ is the initial SoC.

The control of HBC4 is implemented as shown in Fig. 5(b) where the limiter is used to saturate the $\mathrm{SoC}$ to its maximum and minimum values.

According to (1), $v_{i}$ varies based on $S o C_{i}$ of the local BESS in a home. The home with the highest $v_{i}$ supplies the highest power. The home with the lowest $v_{i}$ receives the highest power 
[12]. $v_{i}$ is maximum $\left(v_{i}=V_{\max }\right)$ when the BESS is fully charged during underloads. It is minimum $\left(v_{i}=V_{\min }\right)$ when the BESS is fully discharged during overloads. The voltage, $v_{D C}$ on the DC bus can be expressed in terms of $v_{i}$ as follows

$$
v_{D C}=\sum_{i=1}^{n} \frac{v_{i}}{R_{i}} / \sum_{i=1}^{n} \frac{1}{R_{i}}
$$

where $R_{i} \neq 0$ is a distribution line resistance.

The power exchange on a distribution line is then by voltage difference as follows

$$
P_{g i}=v_{i}\left(v_{D C}-v_{i}\right) / R_{i}
$$

where $P_{g i}$ is the power received by home $i$ (if $P_{g i}>0$ ) and the power supplied to other homes (if $P_{g i}<0$ ).

According to (2), the $v_{D C}$ is maximum when $v_{i}=V_{\max }$ for $i=1, \cdots, n$. That is, when all BESSs in the cluster are completely over charged. Similarly, $v_{D C}$ is minimum when $v_{i}=V_{\min }$ for $i=1, \cdots, n$. That is, when all BESSs in the cluster are completely over discharged. Consequently, $P_{g i}=0$ in (3) for all $i=1, \cdots, n$ when all the BESS in the cluster are either overcharged $\left(v_{D C}=v_{i}=V_{\max }\right)$ or over discharged $\left(v_{D C}=v_{i}=V_{\min }\right)$. Thus, unlike the case with conventional clusters [25], [26], the power balance equation, $\sum_{i=1}^{n} P_{g i}$ evaluated at the DC bus in Fig. 2 cannot be used to determine the amount of power surplus/deficit in the cluster because $\sum_{i=1}^{n} P_{g i}=0$.

The only way to explicitly determine the amount of power surplus/deficit in the cluster shown in Fig. 2 is to obtain the algebraic sum of power flow in the cluster taking the FPC losses and distribution line losses into account. The FPC loss, $P_{\text {Closs }, i}$ can be calculated using the loss model developed in [11]. Fig. 6 shows the accuracy of the loss model for estimating the $P_{\text {Closs }, i}$ and the efficiency of the FPC. The distribution loss, $P_{\text {loss }, i}$ in each line can be calculated as

$$
P_{l o s s, i}=\left(v_{D C}-v_{i}\right)^{2} / R_{i}
$$

Then taking the algebraic sum of power flow in the cluster, the power surplus/deficit, $P_{\text {net }}$ in a cluster can be calculated as

$$
P_{n e t}=\sum_{i=1}^{n}\left(P_{p v, i}+P_{b, i}\right)-\sum_{i=1}^{n}\left(P_{L, i}+P_{\text {Closs }, i}+P_{\text {loss }, i}\right)=0
$$

where $P_{p v, i}$ is the power from the PV, $P_{L, i}$ is the power drawn by the DC loads, $P_{b, i}$ is the power charged by the BESS if $P_{b, i}$ $<0$ and discharged by the BESS otherwise. Based on the sign of $P_{n e t}$, the operating modes of the cluster can be classified into three categories as: normal $\left(P_{n e t}=0\right)$, underload $\left(P_{n e t}>0\right)$ and overload $\left(P_{n e t}<0\right)$ operating modes. However, the determination of $P_{n e t}$ in (5) requires a centralized controller that collects $P_{p v, i}, P_{b, i}, P_{L, i}, P_{\text {Closs }, i}$ and $P_{\text {loss }, i}$ from every home in the cluster to perform the calculation given by (5) from a single central point. This requires a high bandwidth communication especially when the cluster is large. Furthermore, the central controller itself is a source of a single point of failure, imposing a reliability risk to the cluster [28]. Therefore, other indirect methods are required to classify the operation mode of the cluster and determine $P_{\text {net }}$.

\section{Proposed Control Strategy}

This section describes the operation and control of two inter-

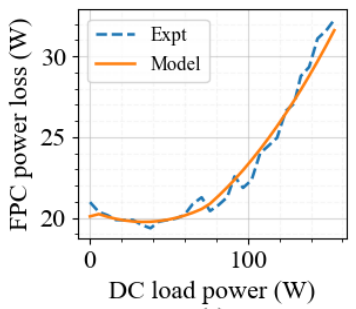

(a)

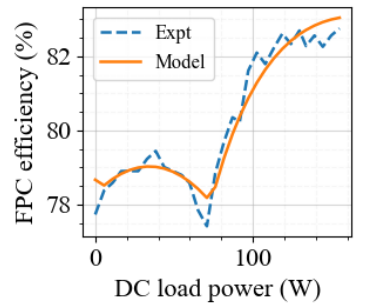

(b)
Fig. 6. Accuracy of the FPC loss model for approximating $P_{\text {Closs }, i}$ at any given operating condition.

connected clusters. Firstly, the operation of the clusters in the interconnected mode is described. Then, the proposed control strategy is presented.

\section{A. Operation of Two Interconnected Clusters}

Fig. 7 shows the schematic of two interconnected clusters; clusters $j$-and $k$ using two ICs; IC- $j$ and IC- $k$. As cited in [29], the cost of power semiconductor devices is decreasing between $1 \%$ and $5 \%$ every year for the same output performance. Converter efficiencies greater than $98 \%$ can also be achieved with advanced modulation strategies [30]. Thus, in the near future, the cost and conversion losses of the ICs will reduce and the interconnection of clusters using two ICs will become economically feasible.

The ICs do not allow bidirectional power flow between the clusters when both clusters have sufficient energy resources to support their PV generation and load demand, i.e. when $P_{n e t}=0$. In this mode, the ICs can be turned off to minimize the power losses in the ICs. However, if one cluster is overloaded $\left(P_{\text {net }}<0\right)$, the power deficit can be imported from the second cluster. Similarly, if one cluster is underloaded $\left(P_{n e t}>0\right)$, the power surplus can be exported to the second cluster. When both clusters are overloaded and underloaded at the same time, no power exchange occurs. Thus, the advantage of interconnecting clusters is to provide power support during periods of underloads and overloads.

However, due to the drawbacks of a central controller, the determination of $P_{n e t}$ which is required to classify the operating mode of a cluster as normal, underload and overload remains a challenge. Moreover, one of the goals of this paper is to reduce cost by operating the clusters without using any communication links. Therefore, the central controller which relies on communication links is not required.

According to (2), $v_{D C}=V_{\max }$ when there is an underload $\left(P_{n e}>0\right)$ in the cluster. Similarly, $v_{D C}=V_{\min }$ when there is an overload $\left(P_{n e t}<0\right)$ in the cluster. Since $V_{\max }$ and $V_{\min }$ are known in advance, this means that the $v_{D C}$ can also be used to indicate the operating mode of the cluster. When $v_{D C}=V_{\max }$ or $v_{D C}=V_{\min }$, the cluster has the maximum absolute value of $P_{\text {net }}$. To this end, the operating modes of the cluster are (re)classified into three categories: normal, underload and overload operating modes based on $v_{D C}$ as shown in Fig. 8. Here, $v_{L}$ and $v_{H}$ are voltage thresholds that are set to explicitly separate the operating modes of the cluster. The $v_{L}$ and the $v_{H}$ voltage thresholds also helps the cluster to transit smoothly into over- 


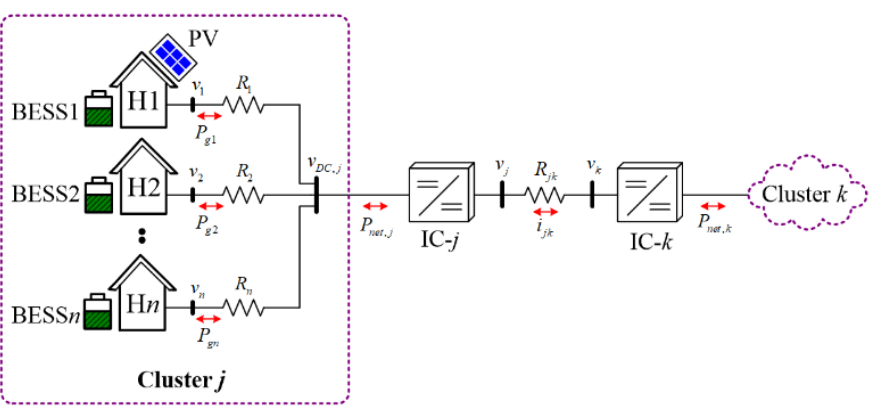

Fig. 7. Interconnection of two clusters $-j$ and $k$ using two bidirectional DCDC converters; IC $-j$ and IC- $k$ and a TL with resistance $R_{j k}$.

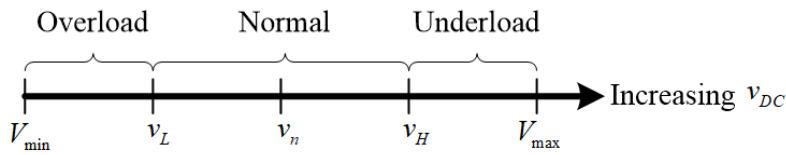

Fig. 8. Operating modes: overload, normal and underload of a cluster based on the DC bus voltage, $v_{D C}$.

load and underload operating modes. Power exchange is triggered when $v_{D C}>v_{H}$ (i.e. cluster is underloaded) and when $v_{D C}<v_{L}$ (i.e. cluster is overloaded). If $v_{L} \leq v_{D C} \leq v_{H}$, the cluster is in the normal operating mode and can only receive power if the neighboring cluster is underloaded. To avoid unnecessary power exchange between the clusters, the normal operating mode has a wider voltage operating range than the overload and underload operating modes.

\section{B. Proposed Control Strategy}

The main objective of this paper is to efficiently coordinate the power exchange between two interconnected clusters while taking TL losses into account. The clusters considered in this paper have unregulated DC bus voltages, $v_{D C}$. The only way to efficiently exchange power between the clusters is through the adjustment of $v_{j}$ and $v_{k}$ at the terminals of IC- $j$ and IC- $k$ respectively; hence the use of two ICs as shown in Fig. 7.

To avoid the use of a communication based centralised controller which can explicitly calculate $P_{\text {net }}$ i.e. the amount of power transfer required by a cluster, a decentralized control strategy is proposed in this paper. The proposed strategy is a combination of a local droop-based control method and a midpoint voltage control method each of which has been used for specific objectives.

The proposed droop control method provides reference voltage values to the ICs in Fig. 7 based on the $v_{D C}$ of each cluster. As a result, the amount of power required by each cluster and the direction of power flow on the transmission line is automatically established.

The mid-point voltage control method corrects the reference voltage values provided by the droop control method in order to reduce the TL loss only when one cluster is operating in a normal operating mode (where it can either import or export power) while the second cluster is either overloaded or underloaded. In other operating conditions, each cluster has a specific power surplus/deficit which needs to be satisfied. As a result, the clusters do not have the flexibility to either import or export power which is required to reduce the TL loss.
Consequently, the mid-point voltage control method is deactivated and the power exchange between the clusters get managed by the droop control method.

\section{a. Local Droop-Based Control Method}

In the local droop-based control method, each cluster measures the corresponding $v_{D C}$ to determine its mode of operation according to Fig. 8. Then the $v_{D C}$ is used in a droop function to determine the suitable TL reference voltage for power exchange. Since each cluster can operate in three different operating modes as shown in Fig. 8, two distinct droop functions are used to determine the TL reference voltage as follows

$$
v_{\gamma}^{r e f}=\left\{\begin{array}{l}
v_{\text {nom }}+\alpha_{H}\left(v_{D C, \gamma}-v_{H}\right) \quad \text { if } v_{D C, \gamma}>v_{H} \\
v_{\text {nom }} \quad \text { else if } v_{L} \leq v_{D C, \gamma} \leq v_{H}, \gamma=[j, k] \\
v_{\text {nom }}+\alpha_{L}\left(v_{D C, \gamma}-v_{L}\right) \quad \text { otherwise }
\end{array}\right.
$$

where, $v_{\text {nom }}$ is the nominal TL voltage, $\alpha_{L}$ is the droop gain in the overload operating mode and $\alpha_{H}$ is the droop gain in the underload operating mode.

The droop gain, $\alpha_{L}$ is calculated as the ratio of maximum deviation of $v_{\gamma}$ to the maximum deviation of $v_{D C, \gamma}$ as follows

$$
\alpha_{L}=\frac{v_{\gamma(\min )}-v_{\text {nom }}}{\left(V_{\min }-v_{L}\right)}
$$

where $v_{\left.\gamma_{\text {min }}\right)}$ and $V_{\min }$ is the minimum TL voltage and DC bus voltage respectively.

Similarly, $\alpha_{H}$ is calculated as follows

$$
\alpha_{H}=\frac{v_{\gamma(\max )}-v_{\text {nom }}}{\left(V_{\max }-v_{H}\right)}
$$

where $v_{\chi_{(\max )}}$ and $V_{\max }$ is the maximum TL voltage and DC bus voltage respectively.

According to (6), if $v_{D C, \gamma}$ exceeds the threshold, $v_{L}$, then $v_{\gamma}^{r e f}$ will be reduced to a value below $v_{\text {nom }}$. That means the cluster is overloaded and imports power from the nearby cluster. For example, if cluster $j$ is overloaded while cluster $k$ is in the normal operating mode and assuming that the measured TL voltages are equal to their reference values (6), then $v_{j}=v_{\text {nom }}+\alpha_{L}\left(v_{D C, j}-v_{L}\right)$ and $v_{k}=v_{\text {nom }}$ respectively. As a result, the power received at the terminals of IC $-j$ is given as

$$
P_{j}=-\alpha_{L}\left(v_{D C, j}-v_{L}\right)\left[v_{n o m}+\alpha_{L}\left(v_{D C, j}-v_{L}\right)\right] / R_{j k}
$$

For simplicity, assume that IC- $j$ is lossless, then at steady state, the power, $P_{\text {net, } j}$ received by cluster $j$ is approximately equal to $P_{j}$, i.e. $P_{n e t, j}=P_{j}$. Due to the injection of $P_{n e t, j}$ into the DC bus of cluster $j$, the DC bus voltage will increase. Taking the direction of $P_{n e t, j}$ into a DC bus as negative, the value of the increased DC bus voltage can be calculated from the algebraic sum of powers injected into the DC bus as follows

$$
v_{D C, j}=\frac{\sum_{i=1}^{n}\left(v_{i} / R_{i}\right) \pm \sqrt{\left(\sum_{i=1}^{n}\left(v_{i} / R_{i}\right)\right)^{2}-4 P_{n e t, j} \sum_{i=1}^{n}\left(1 / R_{i}\right)}}{2 \sum_{i=1}^{n}\left(1 / R_{i}\right)}
$$

Since $v_{D C, j}$ should be equal to the bus voltage calculated using (2) when $P_{n e t, j}=0$, a positive solution ( + ) of (10) is taken and the negative solution (-) is discarded. Then, $P_{n e t, j}$ is distributed 
among the homes according to (3) where $v_{D C}=v_{D C, j}$. The home with the lowest $v_{i}$ (with the highest energy deficit) gets the highest power. The converse is also true. If the received power by the home is not enough to meet the local load demand, the excess demand can be load shed. This is however out of scope in this paper.

Similarly, if $v_{D C, \gamma}$ exceeds the threshold, $v_{H}$, then $v_{\gamma}^{\text {ref }}$ will be increased to a value above $v_{n o m}$. That means the cluster is underloaded and exports power to the nearby cluster. Following the similar analysis carried out in the previous paragraphs, however in this case when cluster $j$ exports power to cluster $k$, the same expression given in (10) can be derived. Here, $P_{n e t, j}>0$, so that the withdrawal of $P_{n e t, j}$ from the DC bus decreases $v_{D C, j}$. Consequently, the homes supply $P_{n e t, j}$ to IC-j according to (3). The home with the highest $v_{i}$ (with the highest energy surplus) supplies the highest power and the home with the lowest $v_{i}$ (with the lowest energy surplus) supplies the lowest power.

If $v_{\gamma}^{r e f}=v_{\text {nom }}$, this indicates that the cluster is in the normal operating mode and does not require power support from the nearby cluster. However, if the nearby cluster is either overloaded or underloaded, it should export and import the power respectively.

The operation of BESSs in each home during the power exchange depends on the amount of power it receives/supplies to the associated distribution line, and the operating conditions of the home. For example, if the BESS in a home is empty and the imported power from the distribution line exceeds the load demand, then the BESS will charge. If the imported power is equal to the load demand, then the BESS will be idle. Similarly, during export, if the PV power after export is equal to the load demand, the BESS will be idle.

Both clusters obey the same laws given in (6), (7), (8), (9) and (10). Thus, if both clusters are either overloaded or underloaded at the same time and depending on their $v_{D C}$, droop control method allows automatic power flow from a cluster with a higher $v_{D C}$ to the other with a lower $v_{D C}$ until both clusters reach the same $v_{D C}$ where the power flow is zero.

However, if one cluster is operating in a normal operating mode while the second cluster is either overloaded or underloaded, the local droop control method can lead to poor voltage regulation, low maximum power transfer and high TL loss during power exchange.

For example, consider the situation where cluster $j$ is operating in a normal operating mode while cluster $k$ is operating in an overload operating mode as depicted in Fig. 9. According to (6), $v_{j}=v_{\text {nom }}$ and $v_{k}=v_{n o m}+\alpha_{L}\left(v_{D C, k}-v_{L}\right)$. Applying Kirchhoff's voltage law to Fig. 7, $v_{k}$ can be rewritten as, $v_{k}=v_{j}$ $i_{j k} R_{j k}$ where $i_{j k}$ is the current flow in the TL. Denote the power received by IC- $k$ as $P_{k}$. Then $P_{k}$ can be expressed as

$$
P_{k}=\frac{v_{j} v_{k}-v_{k}^{2}}{R_{j k}}=i_{j k}\left(v_{n o m}-i_{j k} R_{j k}\right), \quad v_{j}=v_{\text {nom }}
$$

and $v_{k}$ as

$$
v_{k}=0.5\left(v_{j} \pm \sqrt{v_{j}^{2}-4 R_{j k} P_{k}}\right)
$$

In the direction of current shown in Fig. 9, a positive (+) solution of $v_{k}$ is taken. If $P_{k}=0$ in (12) then, $v_{k}=v_{j}=v_{\text {nom. }}$. However, if maximum power, $P_{k(\max )}(13)$ is received by IC- $k$

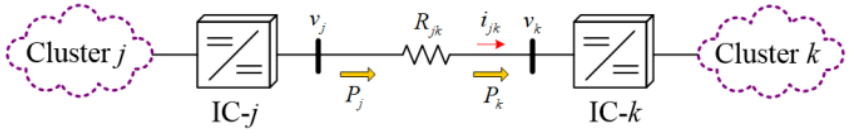

Fig. 9. Interconnection of two clusters $-j$ and $k$ : Cluster $j$ is exporting power to cluster $k$.

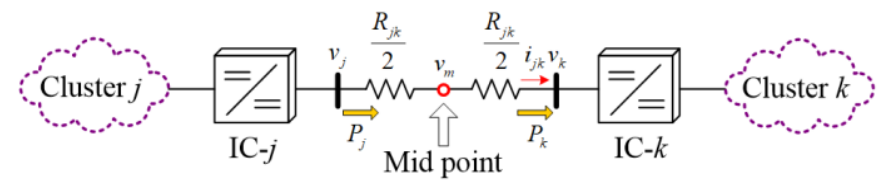

Fig. 10. The concept of the midpoint voltage control method.

$$
P_{k(\max )}=0.25 v_{j}^{2} / R_{j k}
$$

then, $v_{k}=0.5 v_{\text {nom }}$, i.e. the receiving voltage is reduced by $50 \%$ from the nominal voltage. Considering a maximum allowed voltage deviation, $\Delta v_{k(\max )}$ of $\pm 5 \% v_{\text {nom }}$ for most output voltages of power electronic converters [31], this means that $v_{k}$ exceeds the allowed $\Delta v_{k(\max )}=0.05 v_{\text {nom }}$ by $45 \% v_{\text {nom }}$. To maintain the constraint, $\Delta v_{k} \leq \Delta v_{k(\max )}$, the amount of power received by cluster $k$ should be less than or equal to $19 \%$ of $P_{k(\max )}$.

The power loss on the TL can be expressed as

$$
P_{\text {loss }}^{\text {droop }}=\left(0.5 v_{j}-0.5 \sqrt{v_{j}^{2}-4 R_{j k} P_{k}}\right)^{2} / R_{j k}
$$

According to (14), if $P_{k}=0$, then $P_{\text {loss }}^{\text {droop }}=0$. If $P_{k}=P_{\max }$, then $P_{\text {loss }}$ droop $=0.5 P_{\max }$, i.e. $50 \%$ of $P_{\max }$ is power loss in TL.

The above analysis motivates our search for an alternative method to achieve a $P_{k} \geq 0.19 P_{\max }$ when $\Delta v_{k}=\Delta v_{k(\max )}$ and a power loss less than $0.5 P_{\max }$. To this objective, a mid-point voltage control method is proposed.

\section{b. Mid-Point Voltage Control Method}

The mid-point voltage control method only operates to increase the maximum power transfer on the TL and to reduce TL loss when one cluster is operating in a normal operating mode while the second cluster is either overloaded or underloaded. In other operating modes, the clusters operate according to the droop control method given in (6) for the reason described in the opening paragraphs of Section III-B.

Fig. 10 shows the concept of the mid-point voltage control method. In Fig. 10 the TL line is divided into two equal parts and the voltage at the middle of the TL is denoted as $v_{m}$. The idea of the proposed mid-point voltage control method is to keep $v_{m}$ at a constant value during operation. For example, for power to flow from cluster $j$ to cluster $k, v_{j}$ must be increased to a value higher than $v_{m}$ and $v_{k}$ must be decreased to a value lower than $v_{m}$. Effectively, the proposed method allows a power transfer on the TL by varying both $v_{j}$ and $v_{k}$ unlike the proposed local droop control method (6) where the TL voltage is constant for a cluster which is in normal operating mode.

According to Fig. 10, $v_{m}$ estimated from cluster $j$ is given by

$$
v_{m}=v_{j}-0.5 i_{j k} R_{j k}
$$

Similarly, the $v_{m}$ estimated from cluster $k$ is given by

$$
v_{m}=v_{k}+0.5 i_{j k} R_{j k}
$$

Solving (15) and (16) simultaneously, $v_{m}$ can be expressed as

$$
v_{m}=0.5\left(v_{j}+v_{k}\right)
$$

According to (6), $v_{j}=v_{k}=v_{\text {nom }}$ when no power flows on the TL. Substituting $v_{j}=v_{k}=v_{\text {nom }}$ in (17) yields $v_{m}=v_{\text {nom. }}$. Without loss of generalization, $v_{m}$ in Fig. 10 should be controlled to maintain a 
constant $v_{m}=v_{\text {nom }}$ so that a zero-power flow operating condition on the TL is also supported. Therefore, the goal of the proposed mid-point control method is to maintain $v_{m}=v_{\text {nom }}$ by varying $v_{j}$ and $v_{k}$.

Denote the TL voltages adjusted by the mid-point voltage control method as $v_{j(\text { new })}$ and $v_{k(n e w)}$ for cluster $j$ and cluster $k$ respectively. Suppose that the TL is initially open, i.e. $v_{j}=v_{m}=v_{k}=v_{\text {nom }}$. Then, $i_{j k}=0$ and $P_{k}=0$. If the TL is closed with the operating mode depicted in Fig. 10, the relationship, $v_{m}=v_{\text {nom }}$ can be maintained if $v_{j(\text { new })}$ is increased and $v_{k(n e w)}$ decreased by the same amount, $\delta v$ as follows

$$
\begin{aligned}
& v_{j(\text { new })}=v_{m}+\delta v \\
& v_{k(\text { new })}=v_{m}-\delta v
\end{aligned}
$$

Noting that $v_{m}=v_{\text {nom }}$, the new power delivered, $P_{k(\text { new })}$ to cluster $k$ with the proposed mid-point voltage control method can be expressed as

$$
P_{k(\text { new })}=2\left(v_{\text {nom }} \delta v-\delta v^{2}\right) / R_{j k}
$$

Since the power delivered by mid-point voltage control method (20) should be equal to the power delivered by the droop control method (11), $\delta v$ is obtained as follows

$$
\delta v=0.5 v_{n o m} \pm 0.5 \sqrt{v_{n o m}^{2}-2 i_{j k} R_{j k}\left(v_{n o m}-i_{j k} R_{j k}\right)}
$$

Because, $\delta v$ must be equal to zero when $i_{j k}=0$, a negative (-) solution of $\delta v$ is taken. Substituting (21) in (18) and (19), the new reference TL voltages are expressed as

$$
\begin{aligned}
& v_{j(\text { new })}=1.5 v_{\text {nom }}-0.5 \sqrt{v_{\text {nom }}^{2}-2 i_{j k} R_{j k}\left(v_{\text {nom }}-i_{j k} R_{j k}\right)} \\
& v_{k(\text { new })}=0.5 v_{\text {nom }}+0.5 \sqrt{v_{\text {nom }}^{2}-2 i_{j k} R_{j k}\left(v_{\text {nom }}-i_{j k} R_{j k}\right)}
\end{aligned}
$$

In general, the TL voltage of a cluster operating in the normal operating mode is set according to (22), and the TL voltage of a cluster operating in either the overloaded or underloaded operating mode is set according to (23). It then follows that with the proposed mid-point voltage control method, the maximum power received by cluster $k$ is given by

$$
P_{\max }^{\text {new }}=0.5 v_{\text {nom }}^{2} / R_{j k}
$$

The $P_{\max }{ }^{\text {new }}(24)$ is double the value of the maximum received power given by the droop control method in (13). Therefore, by using the proposed method, a higher amount of power can flow on the TL than sorely using the droop control method. Considering the maximum allowed voltage deviation, $\Delta v_{k(\max )}$ of $\pm 5 \% v_{\text {nom }}$ for the TL voltages given in (22) and (23), the maximum amount of received power by cluster $k$ is $38 \%$ of $P_{\max }$, i.e. two times the maximum received power by the droop control method of $19 \%$ of $P_{\max }$. The power loss on the TL with the mid-point voltage control method can be expressed as

$$
P_{\text {loss }}{ }^{\text {midpoint }}=\left(v_{n o m}-\sqrt{v_{n o m}^{2}-2 i_{j k} R_{j k}\left(v_{n o m}-i_{j k} R_{j k}\right)}\right)^{2} / R_{j k}
$$

According to (25), if $P_{\max }$ (13) is substituted in (25), then $P_{\text {loss }}^{\text {midpoint }}=0.34 P_{\max }$, which is $0.15 P_{\max }$ lower than the $P_{\text {loss }}$ droop $=0.5 P_{\max }$ of the droop control method. Fig. 11 shows that the mid-point voltage control method has better voltage regulation and have a lower power loss than the droop control method for the same amount of power delivered to cluster $k$.

\section{Numerical Example}

The reduction of the TL loss when one cluster is operating in the normal operating mode while the second cluster is not can be further illustrated by a numerical example.

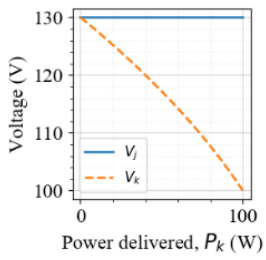

(a)

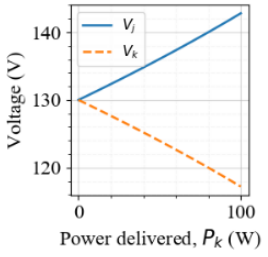

(b)

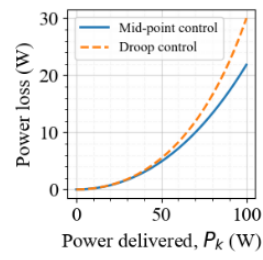

(c)
Fig. 11. Voltage and power loss comparison between the droop control method and the mid-point voltage control method: (a) voltage using droop (b) voltage using mid-point voltage control and (c) power loss comparison.

Consider Fig. 10 where cluster $j$ is operating in the normal operating mode and cluster $k$ is underloaded. Suppose $R_{j k}=0.2$ $p u$ and that cluster $k$ requires $P_{k}=0.5 p u$ to meet its power deficit. Table I summaries the results.

TABLE I

Numerical Example: Reduction of TL loss by the Mid-point Voltage Control Method.

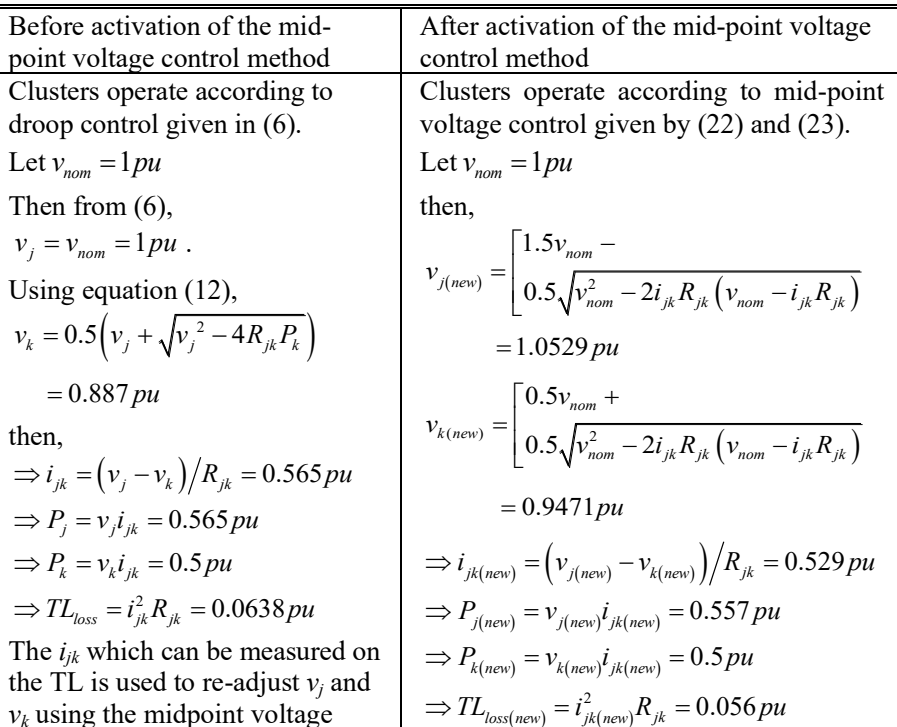
control method which is presented in the right column.

The TL loss is reduced by $12.2 \%$ while maintaining $P_{k}=P_{k(\text { new })}=0.5 \mathrm{pu}$.

\section{CONTROL IMPLEMENTATION}

This section describes the implementation of the proposed droop control and the mid-point voltage control method.

\section{A. Control Implementation}

The purpose of the proposed methods is to provide TL voltage references; $v_{j}^{r e f}$ and $v_{k}^{r e f}$ to IC- $j$ and IC- $k$ respectively. The ICs adjusts $v_{j}$ and $v_{k}$ to follow $v_{j}^{r e f}$ and $v_{k}^{r e f}$ respectively so that a coordinated and efficient power exchange between cluster $j$ and cluster $k$ is achieved. The operation of the midpoint voltage control method in one cluster, however, requires knowledge of $i_{j k}$ on the TL and the operating mode of the second cluster. The $i_{j k}$ on the TL can be locally measured while the operating mode of the second cluster can be known by estimating its TL voltage given that the two equations in (22) and (23) are known to each cluster.

For example, suppose that cluster $j$ is underloaded $\left(v_{D C, j}>v_{H}\right)$ and is exporting power to cluster $k$ in Fig. 9. By measuring $i_{j k}$, cluster $j$ can estimate the operation of cluster $k$ and (re)adjust 


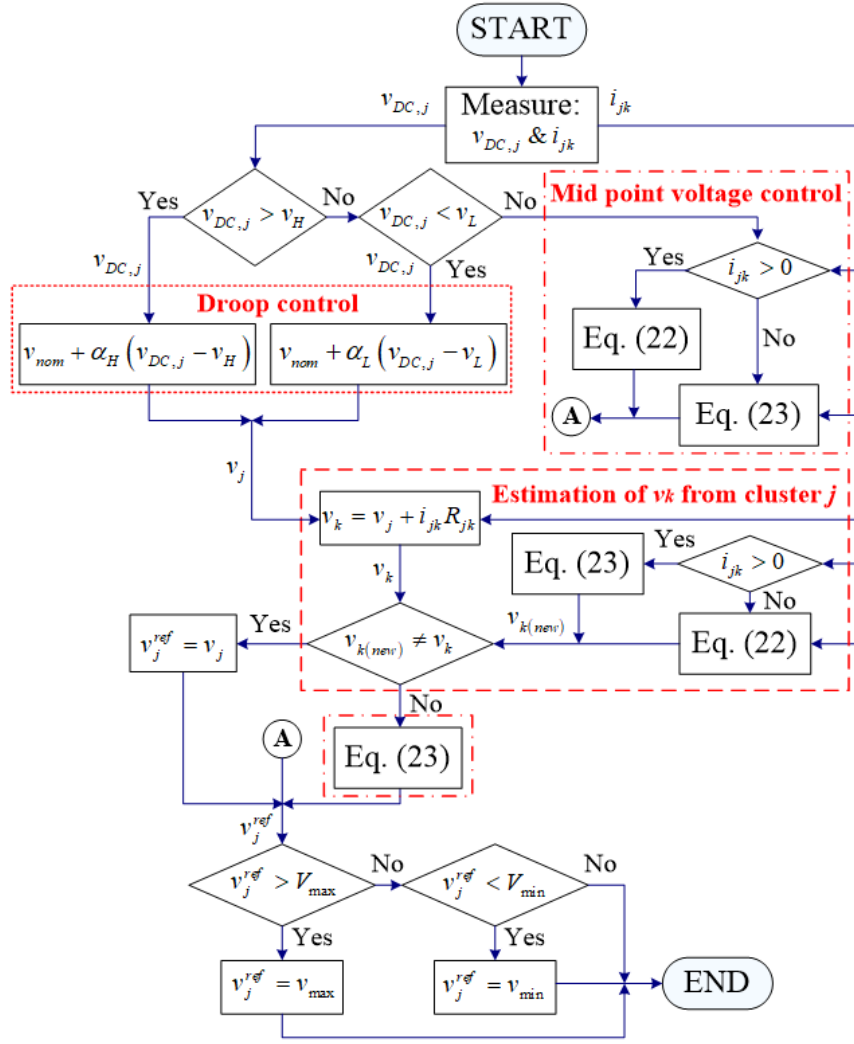

Fig. 12. Flowchart showing the control implementation of the proposed control methods to generate $\mathrm{v}_{j}^{\text {ref }}$ for IC- $j$ in cluster $j$.

its $v_{j}$ which is initially set using droop control (6) as follows.

Firstly, cluster $j$ estimates $v_{k}$ by $v_{k}=v_{j}+i_{j k} R_{j k}$. Secondly, since $i_{j k}$ flowing in the direction shown in Fig. 9 is taken to be positive at cluster $j$ and negative at cluster $k$, cluster $j$ then estimates a new $v_{k}$, i.e. $v_{k \text { (new) }}$ for cluster $k$ by using the expression given in (23). Lastly, cluster $j$ compares $v_{k}$ with

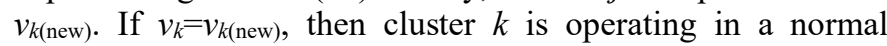
operating mode and $v_{j}$ should be (re)adjusted using the expression given in (23). Otherwise cluster $k$ is either overloaded or underloaded and both clusters should use droop control given in (6) to provide the reference TL voltages. The flowchart in Fig. 12 shows the complete generation of the reference voltage, $v_{j}^{\text {ref }}$ for IC- $j$ in cluster $j$ based on the proposed local droop control method given in (6) and the midpoint voltage control method given in (22) and (23). The same flowchart is also used to provide $v_{k}^{r e f}$ to IC- $k$ in cluster $k$.

\section{B. Control Loops}

The detailed structure of one IC (e.g. IC- $j$ ) is shown in Fig. 13. It consists of two MOSFET switches; $Q_{1}$ and $Q_{2}$ which are switched in complementary, filter capacitors; $C_{d c}$ and $C_{j}$, and filter inductor, $L$. The IC- $j$ is duty cycle, $d$ controlled [11] and adjusts $v_{j}$ to track $v_{j}^{\text {ref }}$ provided by the flowchart in Fig. 12 . When power flows from cluster $j$ to the TL, the IC- $j$ operates in a boost mode. Similarly, when power flows from the TL to cluster $j$, the IC- $j$ operates in a buck mode. During operation, the IC- $j$ has a higher $v_{j}$ than the bus voltage, $v_{D C, j}$.

A cascaded proportional-integral (PI) based dual control loops are used to generate $d$, voltage controller, $G_{v c}$ and current controller, $G_{i c}$ as shown in Fig. 11. $G_{v c}$ has $k_{p, v}$ and $k_{i, v}$ as its PI gains whereas $G_{i c}$ has $k_{p, i}$ and $k_{i, i}$ as its PI gains respe-

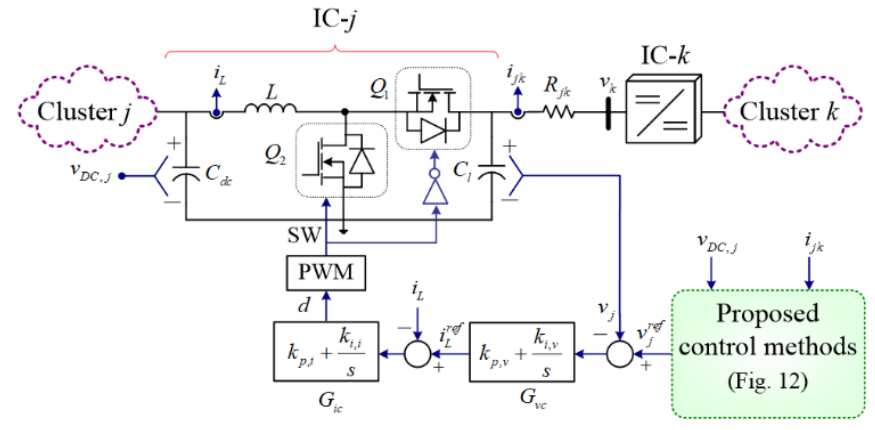

Fig. 13. Structure of IC-j and the associated control loops.

ctively. The purpose of $G_{v c}$ is to adjust $v_{j}$ to follow $v_{j}^{\text {ref. }}$. The input to $G_{v c}$ is the difference between $v_{j}^{\text {ref }}$ and the actual $v_{j}$. The output from $G_{v c}$ is the inductor current reference, $i_{L}{ }^{r e f}$. The main purpose of $G_{i c}$ is to keep the inductor current, $i_{L}$ within the converter current ratings. Thus, the input to $G_{i c}$ is the difference between $i_{L}$ ref and the actual $i_{L}$. The output from $G_{i c}$ is $d$ which is used by the pulse width modulator (PWM) to generate switching signals, SW for IC $-j$. The above description also applies to the control loops of IC- $k$.

\section{Simulation Results AND Discussion}

This section verifies the performance of the proposed control methods. The structure in Fig. 7 was simulated in Matlab/Simulink software. For simplicity, each cluster had three homes with identical BESSs and distribution lines. To avoid running the simulation for long hours due to the complexity of the simulated model, BESSs with small capacities were used. The simulation parameters of the FPC are given in [11] and the other simulation parameters are given in Table II in the Appendix. In the next subsections, the autonomous operation of cluster $j$ and cluster $k$ is presented first, followed by their interconnected operation. To simplify the presentation, cluster $k$ is considered to always operate in the normal operating mode while cluster $j$ can operate in all the three operating modes discussed in Section III.

\section{A. Autonomous Operation of Cluster $j$ and Cluster $k$}

The Fig. 14 shows the autonomous operation of cluster $j$ with step changes in the PV and DC loads in the homes. BESSs are charging if their power output is negative and are discharging if their power output is positive. If the line power is positive, it means a home is exporting power to nearby homes. If the line power is negative, a home is importing power from nearby homes.

As shown in Fig. 14, the homes share their BESS and PV power to meet their load demands. From $0 \mathrm{~s}$ to $80 \mathrm{~s}$, the power from the PV is less that the total load demand in the cluster. As a result, the BESSs in the homes discharge to supply the total load demand in the cluster shown in Fig. 14(d). The BESSs in the homes charge when there is excess power from the PV, i.e. from $80 \mathrm{~s}$ to $180 \mathrm{~s}$. This shows one of the merits of interconnecting homes in a cluster especially in rural areas. The homes tend to share the available energy resources among themselves, making the individual line powers non-zero in Fig. 14. As expected, the total line power in the cluster is zero as shown in Fig. 14 (d) due to conservation of power, i.e. the algebraic sum of line power in the cluster must equal to zero. 
Fig. 15 shows the BESS SoC response in the cluster. As shown in Fig. 15, the power deficit shown in Fig. 14 (d) from $0 \mathrm{~s}$ to $80 \mathrm{~s}$ causes the BESS SoC to decrease. Similarly, the power surplus from $80 \mathrm{~s}$ to $180 \mathrm{~s}$ in Fig. 14 (d) causes the BESS SoC to increase.

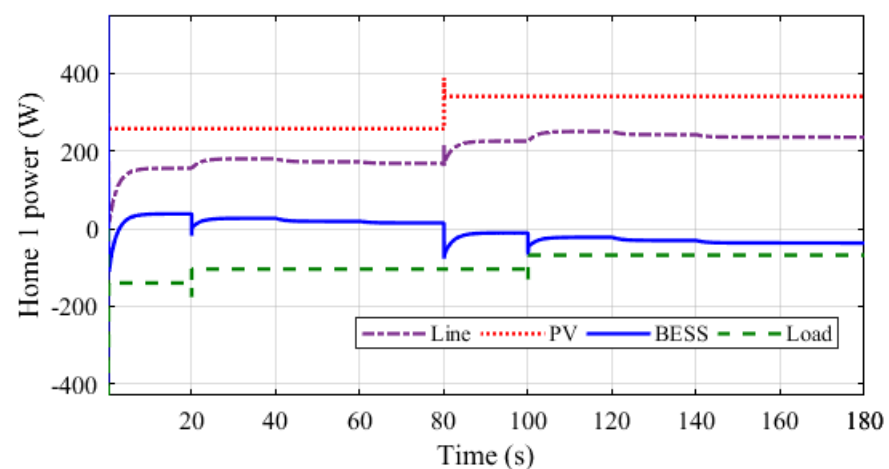

(a)

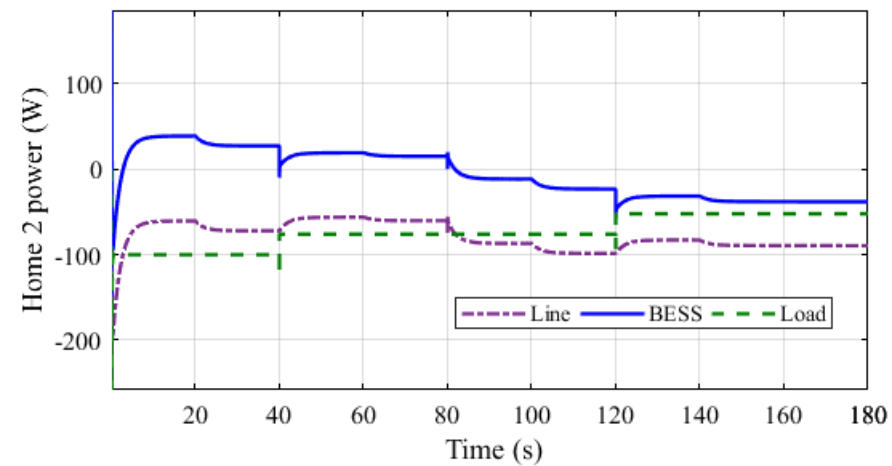

(b)

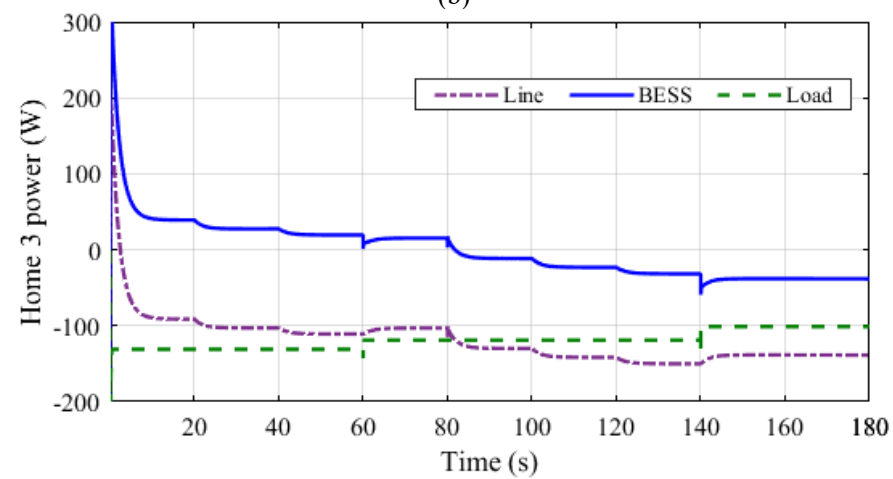

(c)

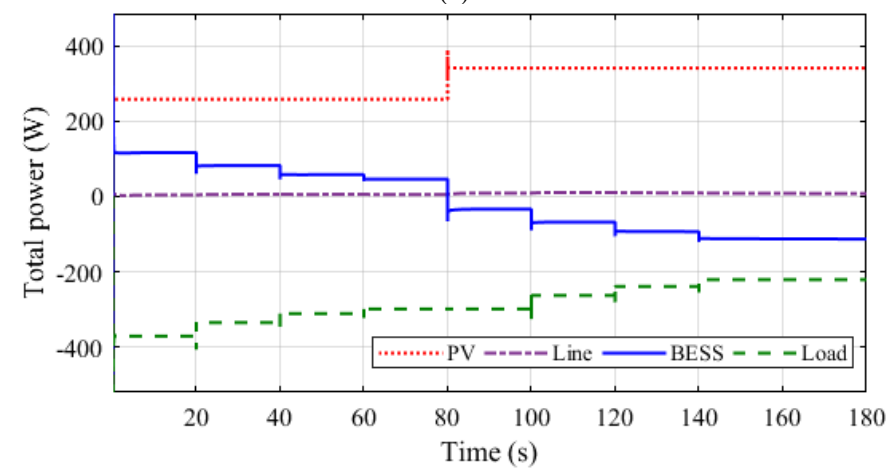

(d)

Fig. 14. Autonomous operation of cluster $j$ : (a) power in home 1 (b) power in home 2, (c) power in home 3 and (d) total PV, line, BESS and load power.

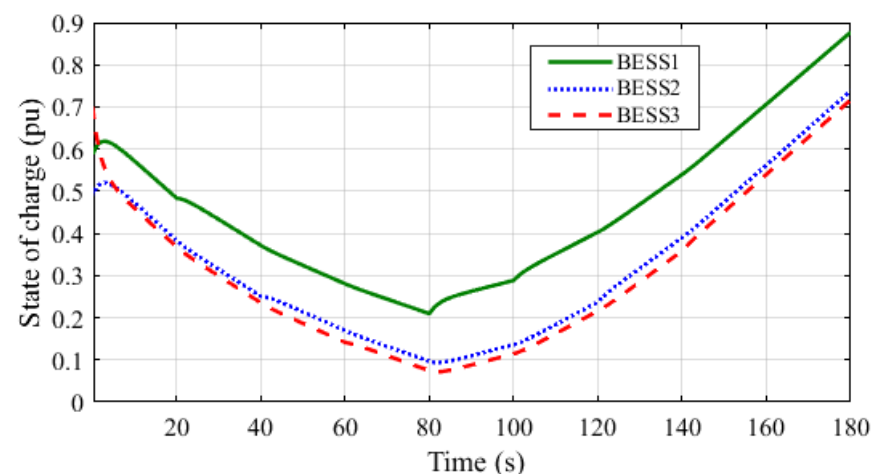

Fig. 15. Autonomous operation of cluster $j$ : BESS SoC response with overcharges $(\mathrm{SoC}>0.8)$ and over discharges $(\mathrm{SoC}<0.2)$.

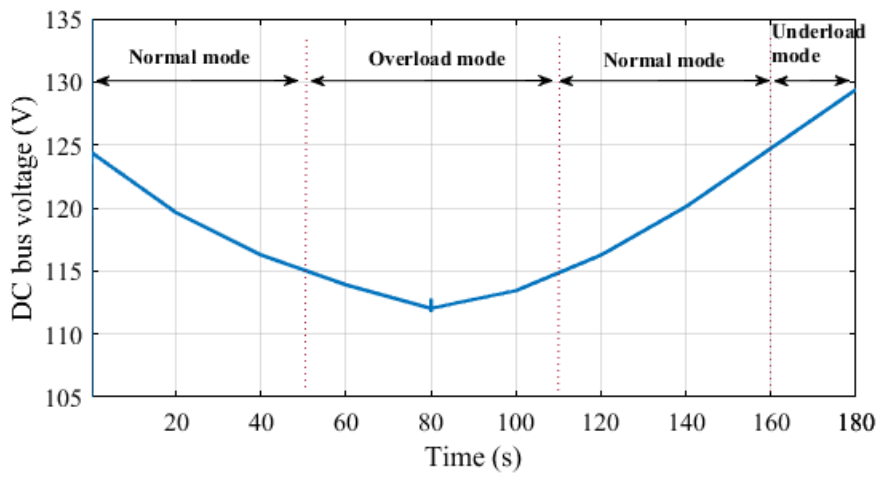

Fig. 16. Autonomous operation of cluster $j$ : DC bus voltage response depicting the three operating modes of a cluster discussed in Section III.

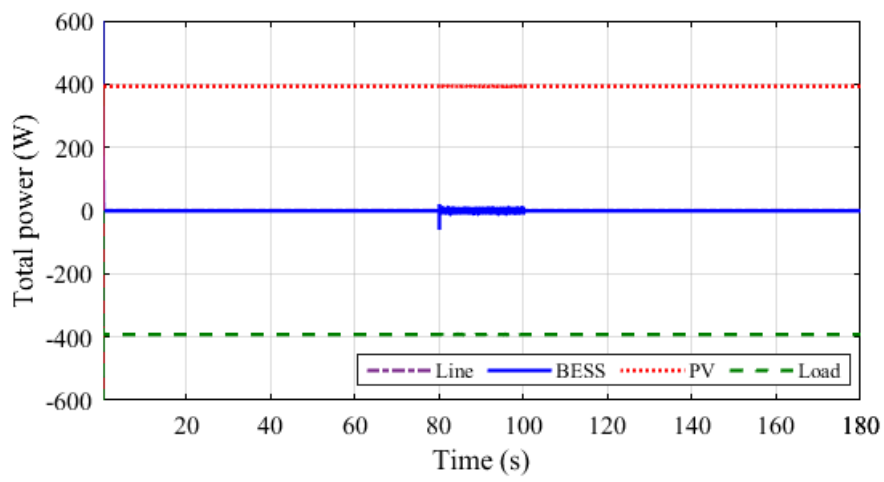

Fig. 17. Autonomous operation of cluster $k$ in the normal operating mode.

Due to lack of an external power source and load to supply the deficit power and absorb the excess power respectively, the BESSs tend to over discharge (i.e. exceeding the minimum $\mathrm{SoC}$ of 0.2 ) and over charge (exceeding the maximum $\mathrm{SoC}$ of 0.8 ) as shown in Fig. 15. This can be avoided by load shedding and PV curtailment techniques. However, this are beyond the current scope.

Fig. 16 shows the variation of the DC bus voltage. According to the DC bus voltage thresholds discussed in Section III, the operation of the cluster is classified as normal, overload and underload operation modes as shown in Fig. 16. Combining Fig. 15 and Fig. 16, we observe that the DC bus voltage gives an indication of the power deficit/surplus extent in the cluster. Here, the overload and under load operating modes cover a bus voltage range of $5 \mathrm{~V}$. For enhanced accuracy, the range can be reduced, e.g. to $2 \mathrm{~V}$ thereby 
increasing the autonomy of the cluster, i.e. the normal operating mode range.

The operation of cluster $k$ in the normal operating mode is shown in Fig. 17. Here, it is assumed that the power from the PV always meets the load demand. Thus, the BESS power output and the distribution line power is zero.

\section{B. Interconnected Operation of Cluster $j$ and Cluster $k$}

Here, the performance of the proposed methods for managing an efficient power exchange between cluster $j$ and cluster $k$ is investigated. To avoid the BESS over discharge and over charge shown in Fig. 15, cluster $j$ should import and export power from/to cluster $k$ during overload and underload operating modes shown in Fig. 16 respectively.

Fig. 18 shows the operation of cluster $j$ when connected to cluster $k$. As shown in Fig. 18 (d), the total line power is no longer zero but is equal to the power that is imported (during the overload period) and power exported (during the underload period) to cluster $k$. During the power import, the BESSs are idle because the imported power is sorely used to supply the deficit load demand. During the export, the BESSs are also idle because, only the surplus PV power is exported to cluster $k$ which is operating in normal operating mode. This shows the suitability of the proposed methods at managing the BESS operation while meeting the load demand in the cluster.

Fig. 19 shows the SoC response of the BESSs in cluster $j$ during the interconnection. Combining Fig. 15 and Fig. 19, we observe that the BESS overcharge and discharge are avoided. This shows the suitability of the proposed method at managing the BESSs in the clusters by coordinating the power exchange between the clusters. Thus, one of the advantages of interconnecting clusters and using the proposed methods is

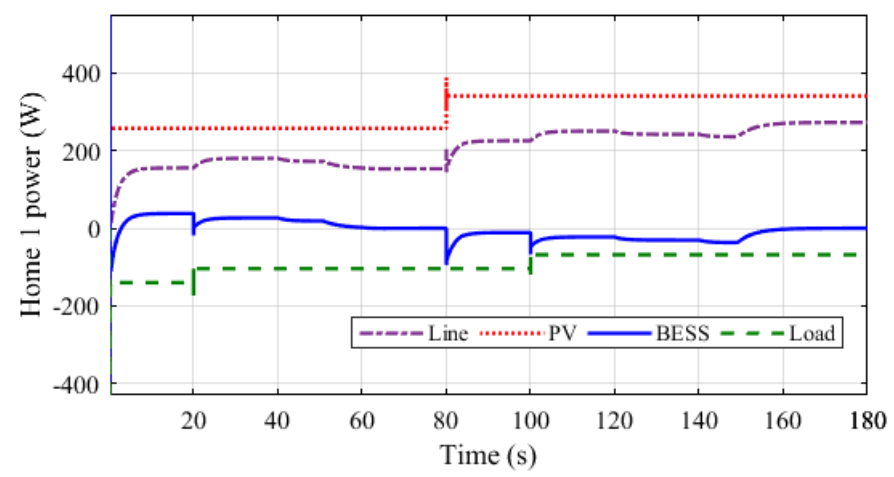

(a)

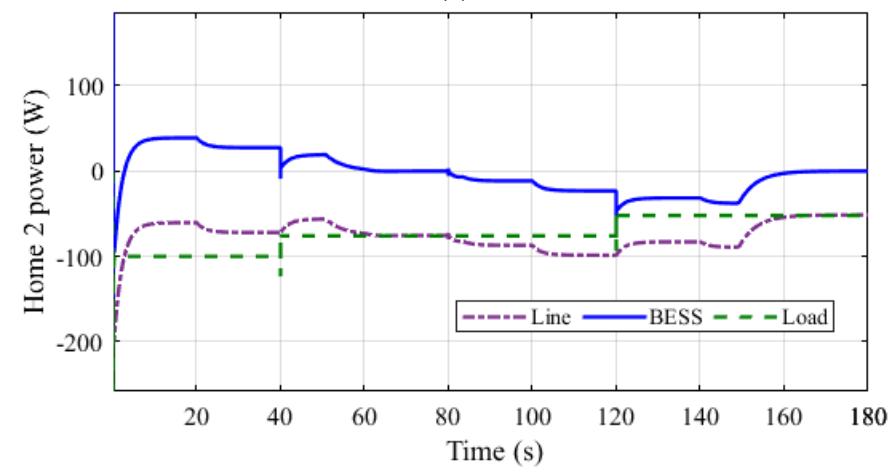

(b)

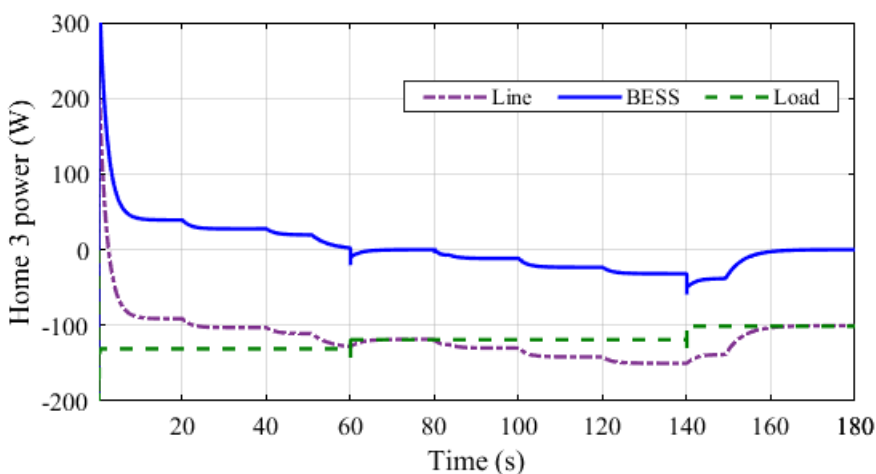

(c)

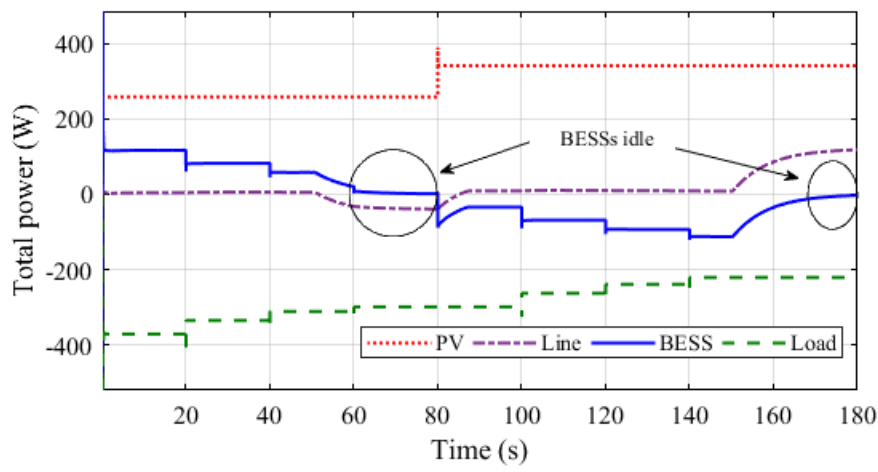

(d)

Fig. 18. Interconnected operation of cluster $j$ : (a) power in home 1 (b) power in home 2, (c) power in home 3 and (d) total PV, line, BESS and load power.

prevention of premature load shedding and PV curtailment when BESSs are overcharged/discharged. However, load shedding and PV curtailment procedures may be required when BESSs in both clusters are overcharged and over discharged. The accuracy to track the minimum and maximum SoC thresholds can be improved by using a small voltage range for the overload/underload operating modes.

Fig. 20 shows the power exchange that takes place between the clusters. As shown in Fig. 20, power is exchanged only when one cluster is either overloaded or underloaded. This ensures autonomous operation of the clusters and avoids unnecessary power flows on the TL which can increase the TL loss. Thus, Fig. 20 illustrates the effectiveness of the proposed methods at coordinating the power exchange between the clusters. Fig. 21 shows the operation of cluster $k$ in the interconnected mode. Due to the operation of cluster $k$ in the normal operating mode, the export/import of power to/from cluster $j$ is used to discharge/charge the BESSs respectively.

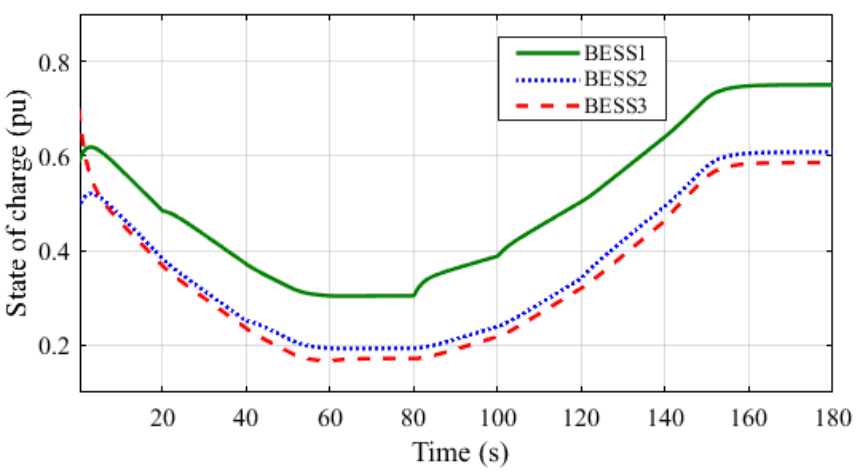

Fig. 19. Interconnected operation of cluster $j$ : BESS SoC response. 


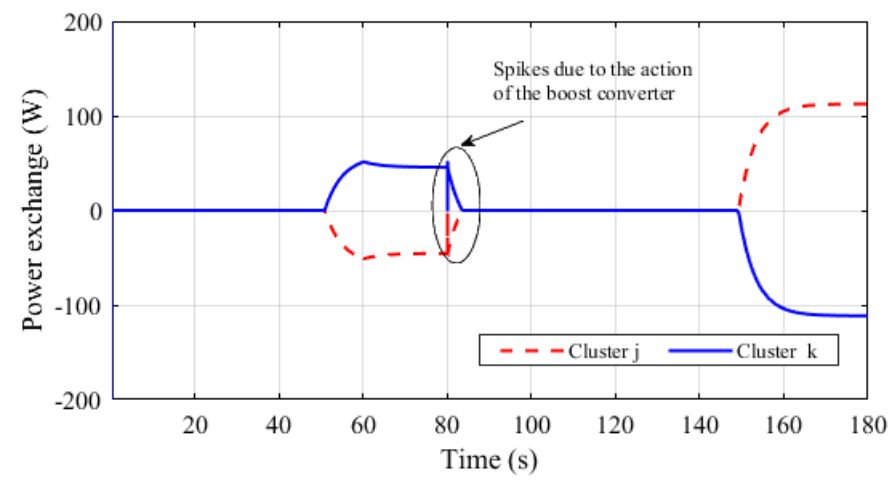

Fig. 20. Power exchange between cluster $j$ and cluster $k$.

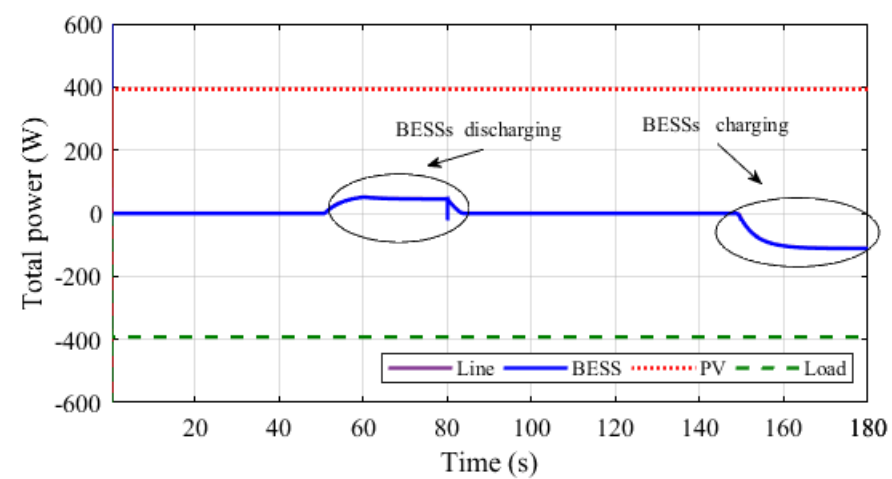

Fig. 21. Interconnected operation of cluster $k$. Due to the operation of cluster $k$ in the normal operating mode, the power export/import is used to discharge/charge the BESS.

\section{Performance of the Mid-point Voltage Control Method}

To illustrate the effectiveness of the mid-point voltage control method, the TL loss comparison with and without the midpoint voltage control method is shown in Fig. 22. As shown in Fig. 22, with the midpoint voltage control method, TL losses are reduced. This also confirms the results shown in Fig. 11(c) and Table I. The magnitude of TL loss depends on the amount of power flow on the TL loss. That is the reason why the TL loss is high when power flow is high in Fig. 20.

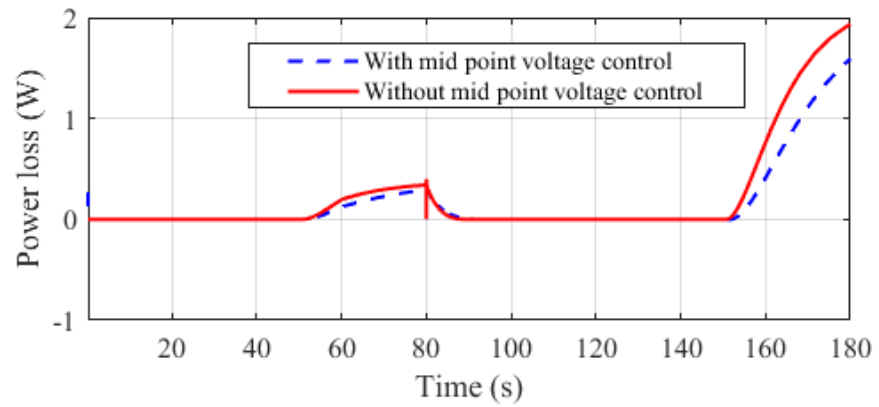

Fig. 22. Power loss comparison when the clusters are operated with and without the virtual midpoint voltage control.

\section{CONCLUSION}

In this paper, two novel decentralized voltage control methods are proposed to efficiently coordinate the power exchange between two interconnected DC clusters which have unregulated DC bus voltages. Two bidirectional DC-DC converters have been proposed to interconnect the clusters in order to take care of the unregulated bus voltages. The simulation results obtained for the interconnected case study show that, the proposed methods can transfer the power between the clusters while managing the BESS operation and meeting the load demand in each cluster. The results also show that the proposed methods can reduce transmission line losses between the clusters where classical optimization techniques cannot be applied. The proposed methods are decentralized and as such, they are suitable for managing the power exchange between the clusters which are designed for energy access. The present work, however, has not considered the stability issues caused by connecting two bidirectional DC-DC converters on the same TL. Thus, the investigation of the stability issues caused by the two converters and the robustness of the proposed methods to faults are a potential future works.

\section{APPENDIX - SIMULATION PARAMETERS}

The parameters used for the simulation study are given in Table II. The parameters used for the FPC have been taken from [11].

TABLE II

Simulation Parameters

\begin{tabular}{clll}
\hline \hline Description & Parameter & Symbol & Value \\
\hline \multirow{2}{*}{ Transmission } & Line resistance & $R_{j k}$ & $4 \Omega$ \\
Line & Nominal voltage & $v_{n o m}$ & $150 \mathrm{~V}$ \\
& Voltage limits & $v_{\max } / v_{\min }$ & $157 \mathrm{~V} / 142 \mathrm{~V}$ \\
\hline \multirow{3}{*}{ DC bus voltage } & Maximum voltage & $v_{\max }$ & $130 \mathrm{~V}$ \\
thresholds & Minimum voltage & $v_{\min }$ & $110 \mathrm{~V}$ \\
& High voltage & $v_{H}$ & $125 \mathrm{~V}$ \\
& Low voltage & $v_{L}$ & $115 \mathrm{~V}$ \\
\hline PI controllers & Voltage controller & $K_{p, r} / k_{i, v}$ & $0.0 / 47$ \\
for interlink & Current controller & $K_{p, i} / k_{i, i}$ & $0.0062 / 326.07$ \\
converters & Droop coefficients & $\alpha_{H} / \alpha_{L}$ & $1.5 / 1.5$ \\
\hline Interlink & Filter capacitance & $C_{l} / C_{d c}$ & $4.8 \mu \mathrm{F} / 3 \mu \mathrm{F}$ \\
converters & Filter inductance & $L$ & $4.8 \mu \mathrm{H}$ \\
\hline & Load voltage/rated power & $V_{L} / P_{\text {rated }}$ & $12 \mathrm{~V} / 150 \mathrm{~W}$ \\
Home in a DC & Distribution line resistance & $R_{i}$ & $1.5 \Omega$ \\
cluster & BESS voltage/capacity & $V_{b} / C_{b}$ & $12 \mathrm{~V} / 0.1 \mathrm{Ah}$ \\
& Maximum SoC & $S o C_{\max }$ & $0.8 \mathrm{pu}$ \\
& Minimum SoC & $S o C_{\min }$ & $0.2 \mathrm{pu}$ \\
& PV voltage/rated power & $V_{p v} / W_{p v}$ & $25 \mathrm{~V} / 400 \mathrm{Wp}$ \\
\hline \hline
\end{tabular}

\section{REFERENCES}

[1] International Energy Agency, 'World Energy Outlook 2019', 2019.

[2] IEA, IREA, WB, and UNSD, 'Tracking SDG7: The Energy Progress Report (2019)', 2019.

African Progress Panel, 'Power People Planet: Seizing Power people planet: seizing Africa's energy and climate opportunities', 2015.

[4] A. Castellano, A. Kendall, M. Nikomarov, T. Swemmer, and B. Africa, 'Brighter Africa: The growth potential of the sub-Saharan electricity sector', 2015.

[5] ESMAP, 'Mini Grids for Half a Billion People: Market Outlook and Handbook for Decision Makers', 2019.

[6] 'Population density (people per sq. km of land area) - Sub-Saharan Africa, Sub-Saharan Africa (excluding high income) | Data'. [Online]. Available: https://data.worldbank.org/indicator/EN.POP.DNST?locations=ZGZF. [Accessed: 30-Mar-2020].

[7] M. M. Hoffmann and D. Ansari, 'Simulating the potential of swarm grids for pre-electrified communities - A case study from Yemen', 
Renew. Sustain. Energy Rev., vol. 108, no. April, pp. 289-302, 2019.

[8] N. Narayan et al., 'Quantifying the Benefits of a Solar Home System-Based DC Microgrid for Rural Electrification', energies, pp. $1-22,2019$.

[9] P. Hollberg, 'Msc thesis: Swarm grids - Innovation in rural electrification', 2015

[10] B. Soltowski, D. Campos-Gaona, S. Strachan, and O. Anaya-Lara, 'Bottom-Up Electrification Introducing New Smart Grids Architecture - Concept Based on Feasibility Studies Conducted in Rwanda', Energies, vol. 12, no. 12, p. 2439, 2019.

[11] C. Samende, N. Mugwisi, D. J. Rogers, E. Chatzinikolaou, F. Gao, and M. Mcculloch, 'Power Loss Analysis of a Multiport DC - DC Converter for DC Grid Applications', in IECON Proceedings (Industrial Electronics Conference), 2018, pp. 1412-1417.

[12] C. Samende, S. M. Bhagavathy, and M. Mcculloch, 'State of Charge Based Droop Control for Coordinated Power Exchange in Low Voltage DC Nanogrids', in IEEE 13th International Conference on Power Electronics and Drive Systems (PEDS), 2019, no. July, pp. $1-6$.

[13] S. Adhikari, Q. Xu, Y. Tang, and P. Wang, 'Decentralized control of DC microgrid clusters', in 2017 IEEE 3rd International Future Energy Electronics Conference and ECCE Asia, IFEEC - ECCE Asia 2017, 2017, pp. 567-572.

[14] Q. Shafiee, T. Dragičević, J. C. Vasquez, and J. M. Guerrero, 'Hierarchical control for multiple DC-microgrids clusters', IEEE Trans. Energy Convers., vol. 29, no. 4, pp. 922-933, 2014.

[15] S. Moayedi and A. Davoudi, 'Distributed Tertiary Control of DC Microgrid Clusters', IEEE Trans. Power Electron., vol. 31, no. 2, pp. 1717-1733, 2016.

[16] M. Mahmoodi, R. Noroozian, G. B. Gharehpetian, and M. Abedi, 'A Suitable Power Transfer Control System for Interconnection Converter of DC Microgrids', in International Conference on Renewable Energies and Power Quality (ICREPQ), 2008, vol. 1, no. $12-14$, pp. $73-79$.

[17] X. Li et al., 'Flexible Interlinking and Coordinated Power Control of Multiple DC Microgrids Clusters', IEEE Trans. Sustain. Energy, vol. 9, no. 2, pp. 904-915, 2018.

[18] Y. Takahashi, K. Natori, and Y. Sato, 'A Multi-Terminal Power Flow Control Method for Next-Generation DC Power Network', in 2015 IEEE Energy Conversion Congress and Exposition (ECCE), 2015, pp. 6223-6230.

[19] H. A. Ramadan, Y. Imamura, and K. Kawachi, 'Multi-level Virtual Conductor Using Bidirectional DC-DC Converters', in Intelec 2013; 35th International Telecommunications Energy Conference, Smart Power and Efficiency, 2013, pp. 105-109.

[20] K. Natori, H. Obara, K. Yoshikawa, B. C. Hiu, and Y. Sato, 'Flexible Power Flow Control for Next-Generation Multi-Terminal DC Power Network', in 2014 IEEE Energy Conversion Congress and Exposition (ECCE), 2014, vol. 2, pp. 778-784.

[21] J. Ma, M. Zhu, X. Cai, and Y. W. Li, 'Configuration and operation of DC microgrid cluster linked through DC-DC converter', Proc. 2016 IEEE 11th Conf. Ind. Electron. Appl. ICIEA 2016, pp. 25652570, 2016.

[22] M. Kumar, S. C. Srivastava, S. N. Singh, and M. Ramamoorty, 'Development of a control strategy for interconnection of islanded direct current microgrids', IET Renew. Power Gener., vol. 9, no. 3, pp. 284-296, 2015.

[23] A. S. Morais, 'Interlink Converters in DC nanogrids and its effect in power sharing using distributed control'.

[24] S. Konar and A. Ghosh, 'Interconnection of Islanded DC microgrids', in 2015 IEEE PES Asia - Pacific Power and Energy Engineering Conference (APPEEC), 2015, vol. 3, pp. 3-7.

[25] U. Vuyyuru, S. Maiti, and C. Chakraborty, 'Active Power Flow Control Between DC Microgrids', IEEE Trans. Smart Grid, vol. 10, no. 5, pp. 5712-5723.

[26] P. Sanjeev, N. P. Padhy, and P. Agarwal, 'Autonomous Power Control and Management between Standalone DC Microgrids', IEEE Trans. Ind. Informatics, vol. 14, no. 7, pp. 2941-2950, 2018.

[27] S. Chun, S. Member, and A. Kwasinski, 'Analysis of Classical Root-Finding Methods Applied to Digital Maximum Power Point Tracking for Sustainable Photovoltaic Energy Generation', IEEE Trans. Power Electron., vol. 26, no. 12, pp. 3730-3743, 2011.

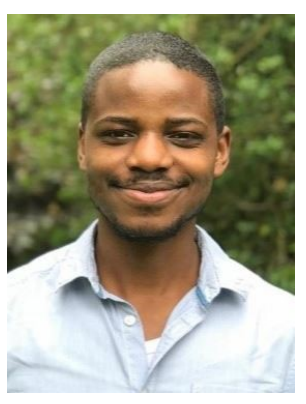

IEEE Trans. Power Electron., vol. 31, no. 2, pp. 1600-1617, 2016. F. Mahmud, 'Toward a new energy paradigm', IEEE Ind. Electron. Mag., vol. 4, no. 1, pp. 52-64.

S. Waffler and J. W. Kolar, 'A Novel Low-Loss Modulation Strategy for High-Power Bidirectional Buck + Boost Converters', IEEE Trans. Power Electron., vol. 24, no. 6, pp. 1589-1599, 2009. S. Sahoo, S. Mishra, and S. Member, 'A Cooperative Adaptive Droop Based Energy Management \& Optimal Voltage Regulation Scheme for DC Microgrids', IEEE Trans. Ind. Electron. (Early Access), pp. 1-11, 2019.

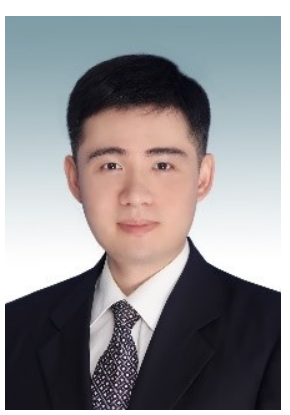

Cephas Samende (M'17) received the B.Eng. degree in electrical engineering from the University of Zambia, Lusaka, Zambia in 2014. In 2016, he joined the University of Oxford, Oxford, U.K, where he is currently pursuing a DPhil degree in Engineering Science in the Energy and Power Group, Department of Engineering Science. His current research interests include modeling and control of power electronics, intelligent control and optimization of networked energy systems designed for energy access in developing countries.

Fei Gao (M'19) received the Ph.D. degree in electrical engineering from the PEMC Research Group, University of Nottingham, Nottingham, U.K., in 2016. From 2016 to 2019, he has been with the Department of Engineering Science, University of Oxford, Oxford, U.K., as a Postdoctoral Researcher.

Since July 2019, he joined Shanghai Jiao Tong University as an associate professor. His current research interests include modeling, control, power management and stability of $\mathrm{dc}$ microgrids and more electric transportation systems. Dr. Gao won the European Union Clean Sky Best PhD Award in 2017 and IET Control \& Automation Runner Up PhD Award in 2018.

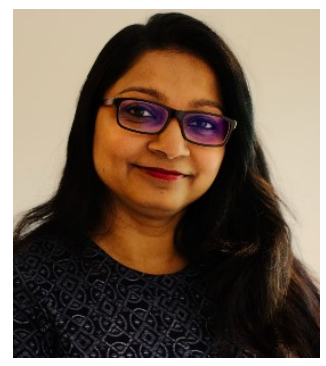

Sivapriya M. Bhagavathy (M'12) received the B.Tech. degree in electrical and electronics Engineering from the University of Kerala in 2006, M.Tech. in energy systems engineering from Indian Institute of Technology, Bombay and the Ph.D. degree in electrical engineering from the Northumbria University in 2018.

She is an Oxford Policy Engagement Network Knowledge Exchange Fellow with the Department of Engineering Science at the University of Oxford, and is a fellow with the Oxford Martin Programme on integrating renewable energy. Her research interests include bridging the gap in energy technology and energy policy, distributed generation and its grid integration, whole system perspective of energy analysis, demand side response using domestic thermal and chemical/electrical storage, electric vehicles as load/storage and electric vehicle infrastructure requirement analysis.

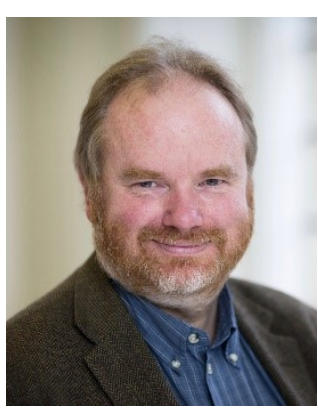

Malcolm McCulloch (SM'89) received the B.Sc. (Eng.) and Ph.D. degrees in electrical engineering from the University of the Witwatersrand, Johannesburg, South Africa, in 1986 and 1990, respectively.

In 1993, he joined the University of Oxford, Oxford, U.K., to head up the Energy and Power Group, where he is currently an Associate Professor in the Department of Engineering Science. He is active in the areas of electrical machines, transport, and smart grids. His work addresses transforming existing power networks, designing new power networks for the developing world, developing new technology for electric vehicles, and developing approaches to integrated mobility. 